\title{
MicroED Structure of Hexagonal Ice Ih
}

Michael W. Martynowycz, Tamir Gonen

Submitted date: 20/06/2019 Posted date: 20/06/2019

Licence: CC BY-NC-ND 4.0

Citation information: Martynowycz, Michael W.; Gonen, Tamir (2019): MicroED Structure of Hexagonal Ice Ih. ChemRxiv. Preprint.

The structure of ice Ih is solved from a single nanocrystal to a resolution of $0.53 \AA \AA$ using the cryoEM method microcrystal electron diffraction (MicroED). Data were collected at just above liquid nitrogen temperatures $(\sim 80 \mathrm{~K})$ in ultra-high vacuum $(\sim 8 \times 10-7 \mathrm{~Pa})$ using a total exposure of less than $1 \mathrm{e}-\AA$ - 2 .

File list (4)

view on ChemRxiv • download file

Figure1.png (3.00 MiB) view on ChemRxiv • download file

Figure2.png (4.13 MiB) view on ChemRxiv • download file table1.png (350.70 KiB) view on ChemRxiv • download file 


\title{
MicroED structure of hexagonal ice Ih
}

\author{
Michael W. Martynowycz ${ }^{1}$ and Tamir Gonen ${ }^{1 \$}$
}

\begin{abstract}
Affiliations
${ }^{1}$ Howard Hughes Medical Institute, Departments of Biological Chemistry and Physiology, University of California, Los Angeles, Los Angeles CA 90095
\end{abstract}

\begin{abstract}
The structure of ice Ih is solved from a single nanocrystal to a resolution of $0.53 \AA$ using the cryoEM method microcrystal electron diffraction (MicroED). Data were collected at just above liquid nitrogen temperatures $(\sim 80 \mathrm{~K})$ in ultra-high vacuum $\left(\sim 8 \times 10^{-7} \mathrm{~Pa}\right)$ using a total exposure of less than $1 \mathrm{e}^{-} \AA^{-2}$. The model has the same unit cell dimensions and space group as structures previously determined by both X-ray and neutron scattering of ice Ih, and obeys the Bernal-Fowler ice rules. Both axial and distal hydrogen densities between oxygen atoms of non-deuterated water ice are resolved. Unaccounted for density between axial hydrogen atoms is observed, and may be a direct observation of a polar bond caused by the electric dipole between oxygen and hydrogen atoms. These observations may have implications for the effects of electron radiation on non-terrestrial ice formations because the conditions experienced by the sample in these experiments are mimetic to those found in near solar space or some planetary bodies without atmospheres, where water ice deposits are exposed to high-energy cosmic rays, thermal stellar emissions, and radiation stemming from solar flares.
\end{abstract}

\$ To whom correspondence should be addressed T.G. (tgonen@g.ucla.edu).

\section{Main text}

The structure and arrangement of oxygen and hydrogen atoms in water, and the arrangement of water molecules in various ice forms, are a topic of great interest in the physical and life sciences(1). Recent analyses of material obtained from space suggest that both amorphous and crystalline ice water deposits exist even under conditions of high vacuum, low temperature, and constant exposure to radiation (2).

There are at least 18 crystalline forms of water ice (3). The simplest and most abundant form on earth is ice $\mathrm{Ih}$, also known as ice one, or hexagonal ice. The atomic arrangement of the oxygen atoms in this form of ice was determined by Barnes in 1929 using X-ray diffraction from single crystals(4). This work laid the foundation for Bernal and Fowler to describe the basic principles governing the arrangement of hydrogen atoms in ice Ih even without direct evidence of hydrogen positions, now known as the BernalFowler ice rules(5). Pauling used these rules of the hydrogen disorder to quantify the residual entropy in ice $\mathrm{Ih}(6)$. Pauling's model suggests each hydrogen/proton lies essentially along the lines that connect adjacent oxygens. Each oxygen may have 4 tetrahedral connections, and each of these hydrogens must then have half occupancy.

Experimental determination of hydrogen positions in ice $\mathrm{lh}$ has proven difficult using X-ray diffraction. To date, most studies of ice Ih have been conducted by powder $\mathrm{X}$-ray diffraction, as large ice crystals tend to form in polycrystalline clumps in a powderlike fashion. X-ray investigations cannot typically resolve individual hydrogen atoms due 
to the inherently weak scattering of the single electron atom. The first electron density map from data collected on a single crystal of ice $\mathrm{Ih}$ from X-ray diffraction was published by Goto et al in 1990(7). This experimental map only demonstrated clear density for the oxygen atoms in the contours. Densities corresponding to hydrogens were found at two positions along the $c$ axis by a difference Fourier synthesis where the contribution from the oxygen atoms was subtracted. These two positions were notably closer to the oxygen than strictly possible for the proton position, and likely correspond to the bonding position of the electron, closer to the heavier oxygen atom.

The hydrogen locations in ice Ih of deuterated water were intensely investigated for decades using neutron diffraction from either powder patterns (8)(Wollen 1949) or single crystal diffraction (9)(Peterson \& Levy 1957). Wollen used powder diffraction that was limited to low resolution, whereas Peterson \& Levy used single crystal neutron diffraction that resolved much higher resolutions. In this way, Peterson \& Levy were able to localize the mean deuterium positions that agreed well with the model suggested by Bernal and Fowler, and ruled out the piezoelectric nature of ice $\mathrm{Ih}$.

While models of ice $\mathrm{Ih}$ were reported from X-ray and neutron diffraction none were reported from electron microscopy. Instead, previous works utilizing electron microscopy either imaged crystals of ice Ih, measured the diffraction intensities from powder rings, or collected data from single orientations of single or polycrystalline material(10). Three dimensional maps generated in electron microscopy correspond to the Coulomb potential of the sample, so such an investigation is highly desirable because it could open an entirely new area of analysis into the science of hydrogen bonding and chemical charges in ice water crystals.

We now report the structure of ice $\mathrm{lh}$ from a single ice nanocrystal to a resolution of $0.53 \AA$ by the cryoEM method microcrystal electron diffraction (MicroED)(11). We measured a single crystal of ice Ih by continuous rotation MicroED(12) using extremely low electron exposures. Ice lh nanocrystals were identified on electron microscopy grids as small, hexagonal crystals with sharp edges (Figure 1). These nanocrystals appeared semi-transparent suggesting they were incredibly thin $(<100 \mathrm{~nm})$. Continuous rotation MicroED (12) data were collected from several nanocrystals in a real space wedge corresponding to $0^{\circ}$ through $+65^{\circ}$. The orientation of every hexagonal ice $\mathrm{lh}$ crystal observed appeared the same, indicating that these crystals preferentially lay on the grid with their basal face perpendicular to the beam direction and prismatic faces parallel to the beam direction. This corresponds to the equivalent $a$ and $b$ axis of the unit cell perpendicular the beam and the $c$ axis being parallel the electron beam at $0^{\circ}$ tilt.

Crystallographic analysis indicated that the unit cell was $(a=b, c)(\AA)=(4.496$, 7.320) with angles $(\alpha=\beta, \gamma)\left(^{\circ}\right)=(90,120)$ in space group $P 63 / \mathrm{mmc}$, or 194 . The data from a single crystal was integrated to a resolution of $0.53 \AA$ with an overall completeness of $65.4 \%, C_{1 / 2}$ of $99.4 \%$ and $R_{\text {meas }}$ of $15.2 \%$. The completeness fell off rapidly between $0.53 \AA$ - the detector corner, and $0.72 \AA$ - the detector edge, where the cumulative completeness was $82 \%$ (Table 1). These dimensions agree well with the previously published values for ice Ih from the literature (13).

The MicroED structure of ice Ih was solved ab initio to a resolution of $0.53 \AA$ (14). The final, refined structure contained a single oxygen atom and two hydrogen atoms as depicted in Figure 1. Though the axial $\mathrm{H}_{1}$ atom was initially located $0.99 \AA$ from the nearest oxygen atom, this $\mathrm{O}-\mathrm{H}$ pair moved considerably from their initial positions during 
refinement, resulting in a final bond length of $1.13 \AA$ and an increase of the O-O distance along $\mathrm{C}$ from $2.76 \AA$ to $2.79 \AA$. The distal $\mathrm{H}_{2}$ atom was placed in a weaker observed density exactly between tetrahedral $\mathrm{O}$ atoms, or approximately $1.37 \AA$ from either oxygen atom. Refinement resulted in this atom moving closer to the nearest $O$ atom to a distance of $1.05 \AA$. This is in line with previously determined structures using neutron diffraction (ref). The typical $\mathrm{H}_{1}-\mathrm{O}-\mathrm{H}_{2}$ molecule formed in this final arrangement would have $\mathrm{H}_{1}$ and $\mathrm{H}_{2}$ atoms at respective distances of $1.13 \AA$ and $1.69 \AA$, and form an $\mathrm{H}_{1}-\mathrm{O}-\mathrm{H}_{2}$ angle of $111.45^{\circ}$, where $\mathrm{H}_{2}$ corresponds to the symmetry equivalent of the nearest $\mathrm{H}_{2}$. The geometrical measures and arrangement of the final structure are presented in Figure 1.

The data show clear, strong density for the oxygen atoms in the unit cell and additional densities for both hydrogen atoms (Figure 2). The strongest difference peak in the Coulomb potential map after fully refining the model is located exactly between the axial oxygens. Similar density at this site was also observed in neutron and X-ray diffraction experiments $(7,9)$. The occupancies of both hydrogen and oxygen atoms within this ice crystal were allowed to vary freely during refinement. The final refinement of the model resulted in the axial $\mathrm{H}_{1}$ atom having $50 \%(1 / 2)$ and the distal $\mathrm{H}_{2}$ atom with an occupancy $8.33 \%(1 / 12)$. The lone oxygen atom also refined to an occupancy of $16.6 \%$ $(1 / 6)$, which we attribute to the inadequacy of scattering factors to account for bonding and charge effects.

Using electrons as a source of radiation in diffraction experiments results in a density map of the Coulomb potential (charge density) in the unit cell, rather than the electron density or scattering length density as determined by X-ray or neutron diffraction, respectively. The Coulomb map shows some interesting features that cannot be observed by other scattering experiments. Specifically, even after modeling and refining the nearest hydrogen positions along the c axis (Figure 2), clear, continuous density was seen both between the hydrogens and oxygen atoms. Additionally, the midpoint between the two oxygen atoms still contains positive density in the difference Fourier map above the $3 \sigma$ level. This is indicative of the model inadequately accounting for the Coulomb potential in this region, and likely caused the modeled atoms along the $c$ axis to move away from their ideal locations consistent with the neutron scattering reports (9).

Indeed, the location directly between the two oxygens is where the Patterson peak was strongest as determined by SHELX(15), indicating that the scattering potential from this region is much stronger than the contribution from the individual atoms alone. We suspect that this density may represent the electrostatic dipole that exists in water molecule. Here, additional Coulomb potential is generated between the slightly electropositive hydrogen atom and the electropositive oxygen atom, resulting in this covalent bond being better described by a "Polar bond." This excess polar potential appears to be present in this data (Figure 2). This density appears directly between the two oxygen molecules, as it would be the summed contribution from both $\mathrm{O}-\mathrm{H}$ dipoles that are partially occupied.

We collected data from multiple hexagonal nanocrystals crystals and attempted to merge these datasets. However all attempts at merging data from different ice nanocrystals failed suggesting that even at the nanoscale these crystalline lattices were non isomorphous. Previous studies suggest that ice crystals may actually contain mixtures between two compatible ice forms, Ih and XI, and may possess multiple stacking faults $(16,17)$. It is possible that each ice crystal contains a unique mixture of stacking 
faults, or mixture of these two forms of crystalline ice providing an atomistic explanation as to why ice crystals in nature (for example snowflakes) adopt different shapes.

Concluding remarks: We report a $0.53 \AA$ resolution structure of ice th from a single nanocrystal determined by MicroED. Investigation of single ice crystals inside a cryoEM at liquid nitrogen temperatures $\left(-193^{\circ} \mathrm{C} /-315^{\circ} \mathrm{F}\right)$ and under $\sim 8 \times 10^{-7} \mathrm{~Pa}$ vacuum allowed us to investigate nanocrystalline ice deposits under conditions that are likely found on extraterrestrial bodies, such as moons, planets distant from their host stars, or in near solar planetary craters surrounding the poles(18). The extraterrestrial ices exist in the ultra-high vacuum of space where they are bombarded by low levels of radiation from thermal electrons produced in the solar corona, electron bursts from solar flares, and cosmic rays - including high energy electrons, that can have velocities similar to those in the a typical TEM $(19,20)$.

Overall the structure agrees well with previous investigations of polycrystalline ice Ih using either scanning or transmission electron microscopy and those from X-ray and neutron scattering investigations. Importantly, the Coulomb maps determined by MicroED indicate that a strong potential is present within the covalent bonds of the ice crystal that could not be directly observed by other methods, and may be indicative of the dipole generated between the oxygen and hydrogen atoms. These crystals display a high degree of non-isomorphism suggesting that no two of these tiny water crystals are alike; and these nanocrystalline seeds may contribute to the polymorphic structures seen in large, natural ice lh crystals. The ability to determine structures of ice from single, nanocrystals by MicroED opens up the possibility of investigating additional forms of ice water that may exist in space, and perhaps even the dynamic processes within water ices under irradiation by extraterrestrial radiation sources.

\section{Acknowledgements}

The Gonen lab is supported by funds provided by the Howard Hughes Medical Institute (HHMI).

\section{Supplementary materials}

A detailed description of the experimental methods, refinement procedures, and CIF model file from this investigation are included in the Supplementary Material for this submission.

\section{Figure and Table legends}

Figure 1. (A) single, hexagonal ice Ih nanocrystal. (B) A representative electron diffraction pattern from a typical ice Ih crystal found in the electron microscope showing high resolution spots and clearly hexagonal diffuse scattering. (C) Geometrical arrangement of the atoms in the unit cell as viewed along the $c$ axis (Top) and $b$ axis (bottom), and (D) the measures of angles and bond lengths along the indicated directions at higher detail.

Figure 2. Coulomb potential map from a single ice Ih nanocrystal along the $(\mathbf{A})$ the $c$ axis, and $(\mathbf{B})$ the $b$ axis. (C) depicts a higher detail map from (B) showing the clear density for the oxygen and hydrogen atoms. Red, dashed circle outlines area of strong density that 
could not be sufficiently modeled using independent atom scattering factors. All maps are $2 \mathrm{~F}_{\mathrm{o}}-\mathrm{F}_{\mathrm{c}}$ maps with volumes colored in wedges of $0.5 \sigma$ level as indicated by the scale bar in the bottom right. Density maps are displayed at XX sigma level.

Table 1. MicroED data collection and modeling statistics for a single ice lh crystal.

\section{References}

1. D. Eisenberg, W. Kauzmann, The Structure and Properties of Water (2007), vol. 9780198570.

2. P. Jenniskens, D. Blake, Structural transitions in amorphous water ice and astrophysical implications. Science (80-. ). 265, 753-756 (1994).

3. M. Millot et al., Nanosecond X-ray diffraction of shock-compressed superionic water ice. Nature. 569, 251-255 (2019).

4. W. H. Barnes, The Crystal Structure of Ice between 0 degrees C. and - 183 degrees C. Proc. R. Soc. A Math. Phys. Eng. Sci. 125, 670-693 (1929).

5. J. D. Bernal, R. H. Fowler, A Theory of Water and lonic Solution, with Particular Reference to Hydrogen and Hydroxyl lons. J. Chem. Phys. 1, 515-548 (1933).

6. L. Pauling, The Structure and Entropy of Ice and of Other Crystals with Some Randomness of Atomic Arrangement. J. Am. Chem. Soc. 57, 2680-2684 (1935).

7. A. Goto, T. Hondoh, S. Mae, The electron density distribution in ice I h determined by single-crystal x-ray diffractometry. J. Chem. Phys. 93, 1412-1417 (1990).

8. E. O. Wollan, W. L. Davidson, C. G. Shull, Neutron Diffraction Study of the Structure of Ice. Phys. Rev. 75, 1348-1352 (1949).

9. S. W. Peterson, H. A. Levy, A single-crystal neutron diffraction study of heavy ice. Acta Crystallogr. 10, 70-76 (1957).

10. A. H. Falls, S. T. Wellinghoff, Y. Talmon, E. L. Thomas, A transmission electron microscopy study of hexagonal ice. J. Mater. Sci. 18, 2752-2764 (1983).

11. D. Shi, B. L. Nannenga, M. G. ladanza, T. Gonen, Three-dimensional electron crystallography of protein microcrystals. Elife. 2013, e01345 (2013).

12. B. L. Nannenga, D. Shi, A. G. W. Leslie, T. Gonen, High-resolution structure determination by continuous-rotation data collection in MicroED. Nat. Methods. 11, 927-930 (2014).

13. K. Röttger, A. Endriss, J. Ihringer, S. Doyle, W. F. Kuhs, Lattice constants and thermal expansion of $\mathrm{H} 2 \mathrm{O}$ and D $2 \mathrm{O}$ ice I h between 10 and $265 \mathrm{~K}$. Acta Crystallogr. Sect. B Struct. Sci. 50, 644-648 (1994).

14. G. M. Sheldrick, Crystal structure refinement with SHELXL. Acta Crystallogr. Sect. C Struct. Chem. 71, 3-8 (2015).

15. G. M. Sheldrick, A short history of SHELX. Acta Crystallogr. Sect. A Found. Crystallogr. 64, 112-122 (2007).

16. T. L. Malkin et al., Stacking disorder in ice I. Phys. Chem. Chem. Phys. 17, 60-76 (2015).

17. S. M. Jackson, V. M. Nield, R. W. Whitworth, M. Oguro, C. C. Wilson, SingleCrystal Neutron Diffraction Studies of the Structure of Ice XI. J. Phys. Chem. B. 101, 6142-6145 (1997).

18. J. R. Spencer, A rough-surface thermophysical model for airless planets. Icarus. 83, 27-38 (1990). 
19. R. P. Lin, Energetic solar electrons in the interplanetary medium. Sol. Phys. 100, 537-561 (1985).

20. J. A. Van Allen, S. M. Krimigis, Impulsive emission of $\sim 40-k e v$ electrons from the Sun. J. Geophys. Res. 70, 5737-5751 (1965).

21. D. Shi et al., The collection of MicroED data for macromolecular crystallography. Nat. Protoc. 11, 895-904 (2016).

22. J. Hattne et al., MicroED data collection and processing. Acta Crystallogr. Sect. A Found. Adv. 71, 353-360 (2015).

23. J. Hattne, D. Shi, M. J. De La Cruz, F. E. Reyes, T. Gonen, Modeling truncated pixel values of faint reflections in MicroED images. J. Appl. Crystallogr. 49, 10291034 (2016).

24. W. Kabsch, Xds. Acta Crystallogr. Sect. D Biol. Crystallogr. 66, 125-132 (2010).

25. G. M. Sheldrick, SHELXT - Integrated space-group and crystal-structure determination. Acta Crystallogr. Sect. A Found. Crystallogr. 71, 3-8 (2015).

26. J. Schindelin et al., Fiji: an open-source platform for biological-image analysis. Nat. Methods. 9, 676-682 (2012).

\section{Supplementary Methods}

Sample preparation. Quantifoil Cu300 R1.2/1.3 holey carbon EM grids were glow discharged for $15 \mathrm{~s}$ and plunged directly into liquid nitrogen using an FEI Vitrobot mark IV at $4^{\circ} \mathrm{C}$ and $100 \%$ humidity.

Grids were clipped and loaded into a Thermo-Fisher Talos Arctica transmission electron microscope equipped with an autoloader held at liquid nitrogen temperatures. The microscope was operated at an accelerating voltage of $200 \mathrm{kV}$, corresponding to a wavelength of $\sim 0.0251 \AA$.

Data collection. Crystals were identified using over-focused diffraction with typical exposure rates less than $10^{-3} \mathrm{e}^{-} \AA^{-2} \mathrm{~s}^{-1}$. Hexagonal crystals selected for data collection were adjusted to eucentric height by adjusting the stage $Z$ such that the crystals remained stationary as the stage was tilted. A select area aperture of $\sim 2 \mu \mathrm{m}$ in projection was inserted to isolate the signal from the crystals.

Continuous rotation MicroED data was collected as previously described(12, 21, 22). Briefly, the stage was continuously rotated at a rate of $0.3^{\circ} \mathrm{s}^{-1}$ while frames from the FEI CetaD camera were read out every $2 \mathrm{~s}$. Data was collected from a real space angular wedge from $+65^{\circ}$ to $0^{\circ}$ using an exposure of $0.003 \mathrm{e}^{-} \AA^{-2} \mathrm{~s}^{-1}$, or a total exposure of approximately $\sim 0.65 \mathrm{e}^{-\AA} \AA^{-2}$.

Data processing. Frames were read out as MRC files and converted to SMV format using software developed in-house and freely available (www.cryoem.ucla.edu/)(23). SMV formatted frames were reduced in XDS(24). XDS initially suggested space group P622 (\#177). Integrated intensities were converted to SHELX format using XDSCONV(15). The structure was solved using SHELXT that placed a single atom into the asymmetric unit in space group $\mathrm{P}_{3} / \mathrm{mmc}(\# 196)(25)$. The initial solution placed the oxygen directly between nearest neighbor oxygen atoms along the $c$ axis with the nearby 
oxygen atoms being strongly present in the $2 \mathrm{~F}_{\mathrm{o}}-\mathrm{F}_{\mathrm{c}}$ and difference maps. The structure was refined by initially moving the original oxygen atom from the midpoint to the axial position along $c$ and refining this single atom solution in SHELXL(14). This solution was refined again allowing the extinction coefficient to be vary. The subsequent solution showed 4 difference peaks greater than the $2 \sigma$ level. The strongest peak corresponded to the midpoint between $c$ axial oxygen atoms. A free hydrogen atom was placed at this location and the structure was refined again. The subsequent refinement showed a continuous density along the $c$ axis between the two oxygen atoms with two difference map peaks split along the bond. The hydrogen was moved to the closest peak within the asymmetric unit and refined again. The strongest difference map peak off the $c$ axis was just off of the tetrahedral axis between the distal $O$ atoms in the unit cell about halfway between the two at $\sim 1.3 \AA$. The final refinement restrained the $\mathrm{O}-\mathrm{H}$ distances to avoid the protons from moving across the asymmetric unit, and keeping them within $1.2 \AA$ from the nearest $\mathrm{O}$ atom. Addition and subsequent refinement of this structure led to the final structure presented in the work, where the axial $\mathrm{H}$ atom moved inward to a distance of $1.05 \AA$. As this position is not occupied when the nearest $O$ is occupied, the symmetry mate would be, placing the atom $\sim 1.7 \AA$ away from the $O$ atom with an angle of $\sim 111^{\circ}$, very near the suggested values of $113^{\circ}$ and $1.68 \AA$ found in other measurements.

Tables and Figures. Figures of atoms and densities were created in PyMol (Schrödinger, 2014). TEM images and diffraction patterns appearing in these figures were contrast and brightness adjusted in $\mathrm{FIJI}(26)$. Overall figures were arranged in PowerPoint (Microsoft, 2018). Tables were created using Excel (Microsoft, 2018). 

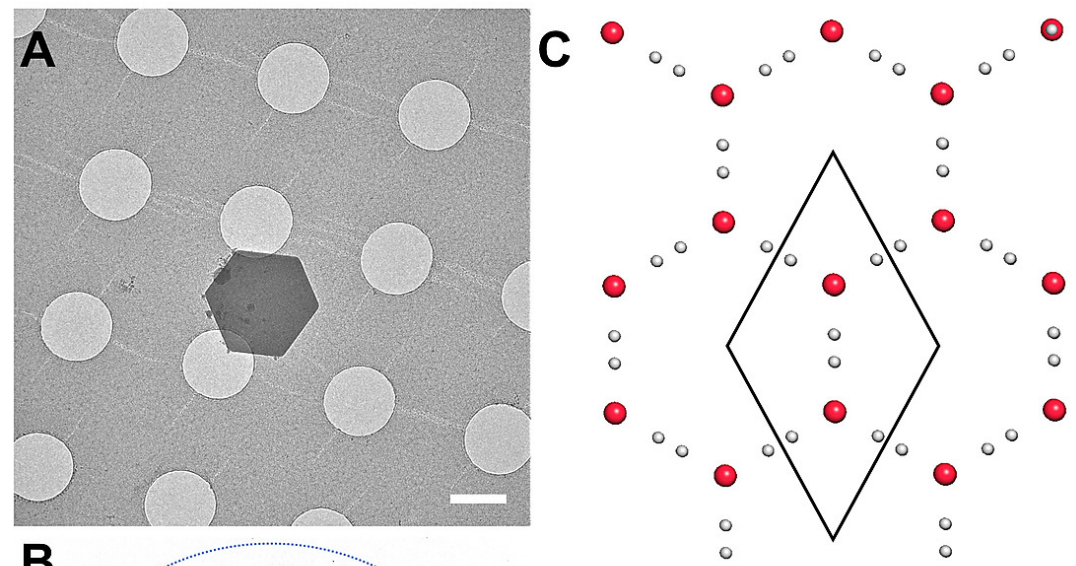

- D $1.05 \AA$
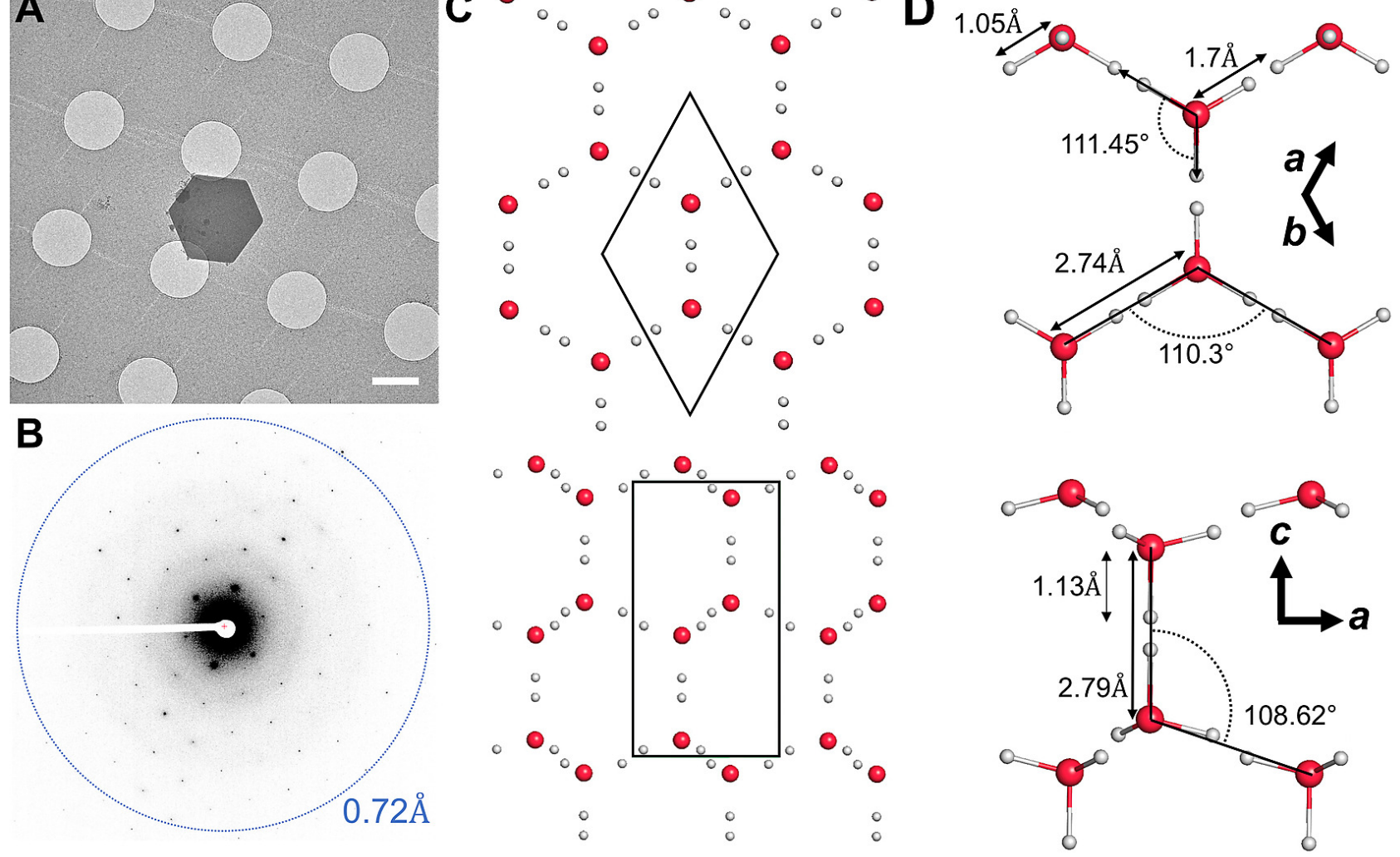

Figure1.png (3.00 MiB)

view on ChemRxiv - download file 


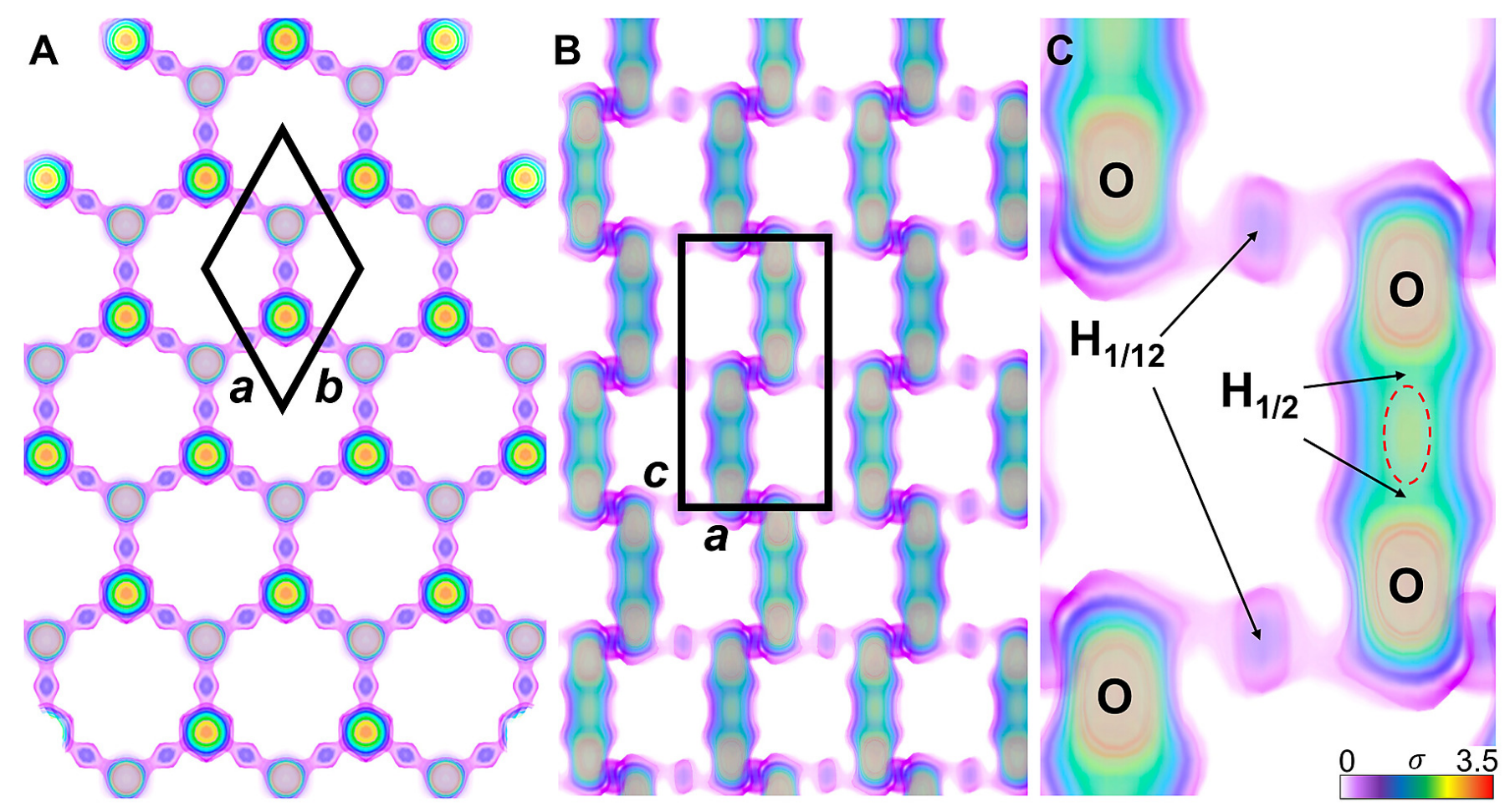

Figure2.png (4.13 MiB)

view on ChemRxiv - download file 


\begin{tabular}{|c|c|c|}
\hline & Parameter & Measure \\
\hline \multirow{8}{*}{ 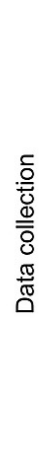 } & Accelerating voltage $(\mathrm{kV})$ & 200 \\
\hline & Electron source & Field emission gun \\
\hline & Total exposure $\left(\mathrm{e}^{-} \AA^{-2}\right)$ & $<1$ \\
\hline & No. of crystals (\#) & 1 \\
\hline & Microscope & $\begin{array}{c}\text { Thermo Fisher Talos } \\
\text { Arctica }\end{array}$ \\
\hline & Detector & CetaD \\
\hline & Rotation rate $\left({ }^{\circ} \mathrm{s}^{-1}\right)$ & 0.3 \\
\hline & Wavelength $(\AA)$ & 0.0251 \\
\hline \multirow{8}{*}{ 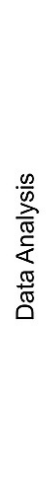 } & Resolution range $(\AA ̊)$ & $7.378-0.53$ \\
\hline & Space group & $\mathrm{P} 6_{3} / \mathrm{mmc}(\# 194)$ \\
\hline & $\begin{array}{l}\text { Unit cell }(a, b, c)(\AA) \\
\left.(\alpha, \beta, \gamma)()^{\circ}\right)\end{array}$ & $\begin{array}{l}4.496,7.320 \\
90.00,120.00\end{array}$ \\
\hline & No. reflections / unique (\#) & $668 / 134$ \\
\hline & $\begin{array}{l}\text { Completeness } \\
(1.0 / 0.72 / 0.53 \text { / overall) }(\%)\end{array}$ & $81.6 / 84.3 / 51.7 / 65.4$ \\
\hline & $\mathrm{R}_{\text {meas }}(\%)$ & $15.2(47.9)$ \\
\hline & $\mathrm{CC}_{1 / 2}(\%)$ & $99.4(79.1)$ \\
\hline & R1 (\%) / wR2 (\%) / GooF & $17.96 / 37.29 / 1.92$ \\
\hline
\end{tabular}

